\title{
Investigation of human chromosome polymorphisms by scanning electron microscopy
}

\author{
CHRISTINE J HARRISON*, ELSPETHM JACK $\dagger$, TERENCE D ALLEN , AND \\ RODNEYHARRIS †
}

From * the Department of Cell Biology and Cytogenetics, and $¥$ the Department of Ultrastructure, Paterson Laboratories, Christie Hospital and Holt Radium Institute, Manchester M20 9BX; and the Department of Medical Genetics, St Mary's Hospital, Manchester M13 OJH.

SUMMARY Human chromosome polymorphisms were investigated by scanning electron microscopy (SEM). Centromeric heterochromatin was of a constricted morphology. The extent of the $\mathrm{C}$ banded region was demarcated by a prominent circumferential groove in $\mathrm{G}$ banded chromosomes. Circumferential grooves were observed within the heterochromatin of chromosome 9 , and the number of grooves present reflected the size of the region. Three dimensional viewing of satellites and short arms of acrocentric chromosomes, from different angles in the SEM, provided the opportunity for accurate assessment of the size of satellites to be made. Also, small morphological variations were defined in the SEM when definition was uncertain in the light microscope (LM).

Chromosome polymorphisms are structural variants of chromosomes displayed most readily at specific chromosome segments, including the highly variable centromeric regions of chromosomes $1,9,16$, the distal end of the $\mathrm{Y}$ chromosome, and the short arms and satellites of acrocentric chromosomes. These regions show a wide variation in size and fluorescent characteristics between persons, and numerous inversions have also been described. ${ }^{12}$ They are generally stable, ${ }^{3}$ present in all cells in a person, and inherited from one generation to the next in a Mendelian fashion. ${ }^{4}$ Therefore, they have been shown to be informative genetic markers, for example, in establishing parental origin of chromosome abnormalities, ${ }^{67}$ particularly in the origin of the extra chromosome 21 in Down's syndrome, ${ }^{8-12}$ and for exclusion of paternity in cases of disputed parentage. ${ }^{1314}$

These chromosome polymorphisms appear to have no direct effect on phenotype, although numerous investigators have sought to discover a role for the frequently observed variations, for example, that variants may produce greater instability of the genome, leading ultimately to an association with clinical abnormality ${ }^{15-20}$ or to an increased risk of developing a malignancy. ${ }^{21-24}$

In light of these suggestions methods to provide reliable comparisons between polymorphisms are

Received for publication 23 May 1984.

Accepted for publication 30 May 1984. essential. Such comparisons produce problems, even within one study, since techniques vary between laboratories. Numerous statistical methods have been used in attempts to overcome these problems (reviewed in Erdtmann ${ }^{25}$ ), but these do not appear to be the answer. We present a new method for direct observation of the polymorphisms using scanning electron microscopy (SEM), which eliminates some of the inconsistencies of the technical procedures and permits direct quantitative assessments to be made.

\section{Materials and methods}

Heparinised peripheral blood was cultured in RPMI 1640 medium (Gibco, Scotland) supplemented with $\rightarrow$ $10 \%$ fetal bovine serum for 72 hours at $37^{\circ} \mathrm{C}$. Then, $0 \cdot 01 \mu \mathrm{g} / \mathrm{ml}$ colcemid (Gibco, Scotland) was added N for 1.5 hours. Cells were incubated in $75 \mathrm{mmol} / \mathrm{l} \mathrm{KCl}$ for 8 minutes at room temperature followed by three washes in methanol:acetic acid fixative (3:1). Chromosome spreads were made by dropping suspension onto coverslips followed by air drying.

Coverslips from those samples showing cen- $\mathbb{D}$ tromeric or Y chromosome polymorphism were $\mathrm{C} \stackrel{\mathcal{P}}{\rightarrow}$ banded according to the method of Sumner ${ }^{26}$ using barium hydroxide and $2 \times \mathrm{SSC}$ at $60^{\circ} \mathrm{C}$, followed by staining in $2 \%$ Giemsa (R66, Hopkins and Williams) in Gurr's buffer ( $\mathrm{pH} \mathrm{6.8)} \mathrm{(Searle,} \mathrm{Bucks).} \mathrm{Cases}$ showing polymorphism of the satellites of the 
acrocentric chromosomes were Giemsa stained only.

Direct comparison of metaphases by light microscopy (LM) and SEM was carried out on G banded specimens, prepared directly in parallel. A modification of the Seabright ${ }^{27}$ technique was used, in which coverslips were incubated in $0.025 \%$ trypsin (Wellcome, Beckenham) in $0.85 \%$ saline for 20 to 40 seconds at $37^{\circ} \mathrm{C}$, followed by Giemsa staining, as above. In some cases it was possible to examine successfully the same coverslip in the SEM after photography in the LM.

$G$ banded specimens for SEM were fixed in $3 \%$ glutaraldehyde (GDA) (Polaron Equipment Ltd, Watford) in $0.1 \mathrm{~mol} / 1$ Sørensen's phosphate buffer $(\mathrm{pH} 7 \cdot 4)^{28}$ for a minimum of 30 minutes. These coverslips were processed for SEM by an osmium tetroxide-thiocarbohydrazide (osmium-TCH) fixation, described in detail elsewhere. ${ }^{29}$ Briefly, specimens removed from GDA were rinsed in Sørensen's buffer and treated as follows: (1) fixation in $1 \%$ osmium in the same buffer for 10 minutes; (2) three rinses in distilled water; (3) 5 minutes' incubation in a freshly prepared saturated solution of $\mathrm{TCH}$ in distilled water; (4) three further rinses in distilled water; and (5) additional fixation for 10 minutes in $1 \%$ osmium in distilled water. Steps (2) to (5) were repeated. The coverslips were then dehydrated through a graded acetone series $(20 \%$ to $100 \%)$, then critical point dried from liquid carbon dioxide with absolute acetone as the transitional fluid. Metaphases were examined in a Cambridge S4-10 stereoscan and an ISI SS 40 electron microscope.

\section{Results}

CENTROMERIC AND Y CHROMOSOME POLYMORPHISMS

Suitable preparations from persons with polymorphisms at the sites of paracentric secondary constrictions of chromosomes 1,9 , and 16, and the distal portion of the $\mathrm{Y}$ chromosome, were initially selected by examination of $\mathrm{C}$ banded metaphases in the $\mathrm{LM}$. The $\mathrm{C}$ banding technique selectively stains constitutive heterochromatin, exhibited in larger amounts at these locations. More detailed investigations of these polymorphic chromosomes were carried out on $G$ banded metaphases, prepared directly in parallel for LM and SEM, using techniques previously described. ${ }^{29} 30$

\section{Chromosome 1}

A subject with an enlarged paracentric secondary constriction in one chromosome $1(1 \mathrm{qh}+)$ was detected by examination of $\mathrm{C}$ banded preparations in the LM. The constriction stained positively with the $\mathrm{C}$ banding technique in all metaphases examined. This demonstrated the presence of almost double the amount of heterochromatin within the centromeric region of the $1 \mathrm{gh}+$ chromosome when compared to the normal number 1 (fig $1 \mathrm{a}$ and $b$, lower insets).

The heterochromatin of the chromosomes 1 stained positively in the $\mathrm{LM}$ after $\mathrm{G}$ banding (fig 1 , upper insets). A pronounced $G$ positive band was observed at the distal boundary of the variable band 1q12 (ISCN 1981 ${ }^{31}$ ) (fig 1b, upper inset, small black arrowhead) corresponding to the limit of the heterochromatic region. When parallel $G$ banded preparations were examined in the SEM this pronounced $G$ band correlated in position to a prominent circumferential groove (fig $1 \mathrm{~b}$, large black arrowhead). This finding supported previous observations of a relationship between LM G bands and the circumferential grooves between the gyres of the quaternary coils of metaphase chromosomes, when viewed in the SEM. ${ }^{29}$

The heterochromatic region of the $1 \mathrm{qh}+$, between the groove and the centromere, was of constricted morphology when compared to the euchromatin. An equivalent, although less prominent, groove was observed in the normal chromosome 1, closer to the centromere (fig 1a, small white arrowhead). This groove also indicated the limit of the heterochromatic region.

\section{Chromosome 9}

Chromosome 9 is the only chromosome of the $\mathrm{C}$ group which exhibits frequent and characteristic polymorphisms. The paracentric secondary constriction at the proximal long arm often appears highly constricted in the LM. This feature has also been demonstrated in the SEM. Fig 2 a shows a chromosome 9 viewed upside down in the SEM. From this position the constricted nature of the centromeric region was demonstrated most clearly.

The $\mathrm{C}$ banding technique revealed this region as a dense heterochromatic block showing considerable variation between persons. Fig $2 b$ and $c$ (upper insets) show a homologous pair of $\mathrm{C}$ banded chromosomes 9 in the LM, of which one member exhibits an increased amount of heterochromatin at the centromere $(9 \mathrm{qh}+)$ (fig $2 \mathrm{~b}$, upper inset), when compared to the other chromosome 9 (fig 2c, upper inset). The heterochromatic secondary constriction of the chromosomes 9 remained pale and rather poorly stained in all $G$ banded metaphases examined in the LM (fig $2 b$ and c, lower insets).

After $G$ banding and examination in the LM, fortuitously, the same metaphase was relocated in the SEM. This enabled a direct comparison to be made of the homologous chromosomes 9 by 


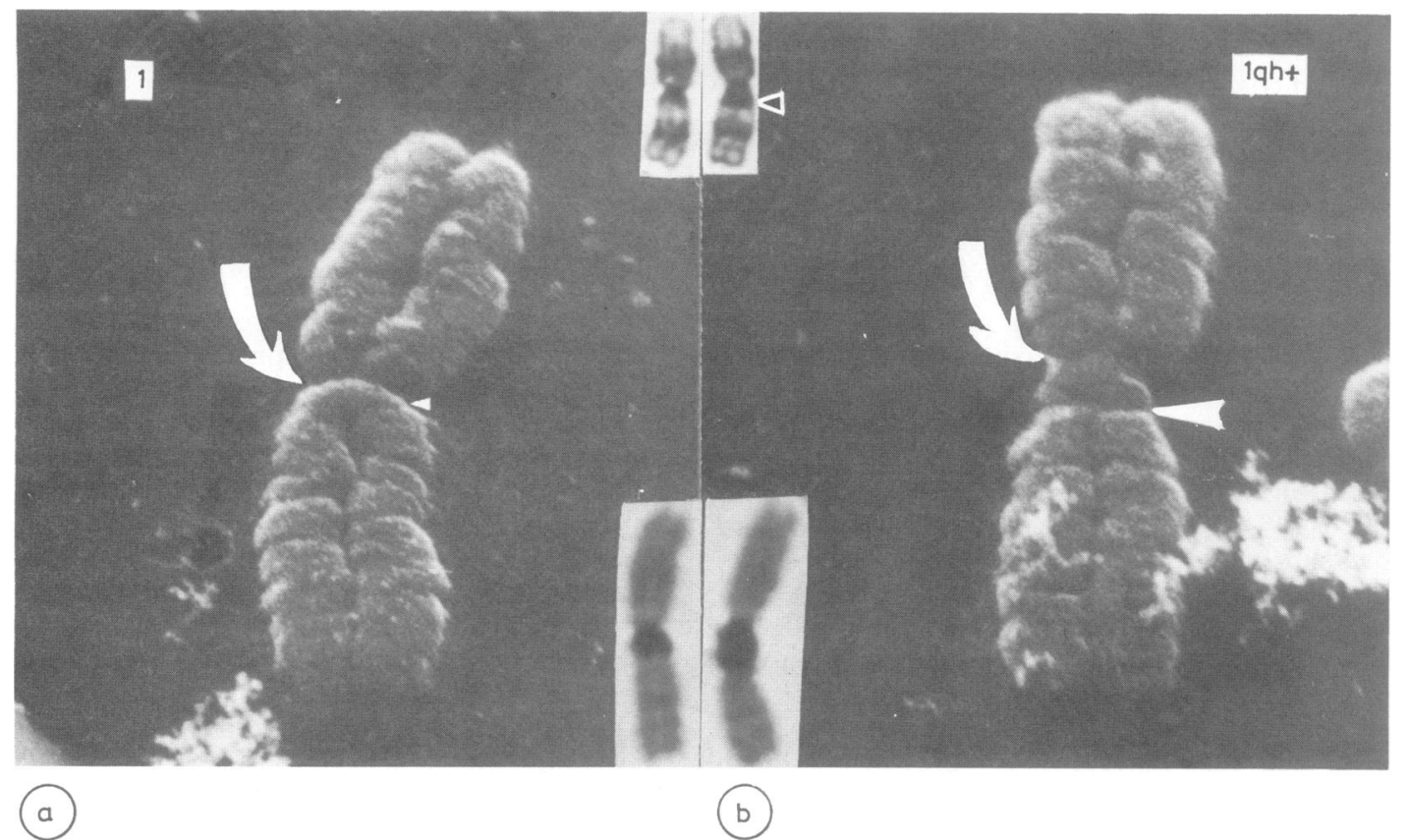

FIG 1 A homologous pair of chromosomes 1 in the SEM, shown with insets of $G$ banded (upper insets) and $C$ banded specimens (lower insets) prepared in parallel. The lqh+ (b) shows approximately twice the amount of heterochromatin within the centromeric region compared to the normal 1 (a). (a) A small groove (small white arrowhead) equivalent to the prominent groove (large white arrowhead $(b))$ is observed closer to the centromere (curved arrow) demarcating the extent of the centromeric region of this chromosome. (b) A pronounced $G$ positive band is observed at the distal boundary of $1 q 12$ demarcating the extent of the $C$ banded region (small black arrowhead) and corresponding in position to a prominent circumferential groove (large white arrowhead). The region between this groove and the centromere (curved arrow) is constricted in morphology. (Original magnification $\times 9000$.)

sequential LM and SEM photography. In these LM photographs the extent of the secondary constriction was demarcated by the proximal boundary of $G$ band $9 \mathrm{q} 21^{31}$ (fig $2 \mathrm{~b}$ and c, black arrowheads, lower insets) which was demonstrated to correlate with a prominent circumferential groove in both homologues in the SEM (fig $2 \mathrm{~b}$ and $\mathrm{c}$, white arrows). The centromere was more clearly defined in the SEM (fig $2 \mathrm{~b}$ and $\mathrm{c}$, black on white arrowheads) than the LM (insets). Therefore, in the SEM the extent of the heterochromatic region between the centromere and the boundary of the secondary constriction were precisely defined, compared to the reduced definition provided by LM of $\mathrm{C}$ or $\mathrm{G}$ banded preparations.

Also, when observed in the SEM, both chromosomes 9 showed additional circumferential grooves within the heterochromatic region. The $9 \mathrm{qh}+$ showed two grooves within this region (fig $2 b$, white arrowheads) compared to the normal homologue, in which only one groove was observed (fig 2c, white arrowhead). This distribution of grooves was consis- tently observed in numerous metaphases from this person. No corresponding $G$ banding pattern was:observed in the metaphases in the LM (fig $2 \mathrm{~b}$ and c, 3 see insets). Therefore the unique observation of variable numbers of intraheterochromatin grooves in the SEM may be used as a reliable evaluation of the relative amounts of heterochromatin in homolo- -5 gous pairs of chromosome 9 . To date these grooves $\frac{7}{0}$ have not been observed in polymorphic chromosomes 1 or 16 .

A pericentric inversion of the heterochromatin of one chromosome 9 (inv (9)) was observed in aO normal subject. This was detected readily by $\mathrm{C \omega}$ banding in the LM (fig $2 \mathrm{~d}$, upper inset). After $\mathrm{G}^{\mathrm{O}}$ banding, the heterochromatin remained poorlyo stained when examined in the LM, even when the euchromatin stained very heavily (fig $2 \mathrm{~d}$, lower ${ }^{\text {? }}$ inset). In this case also the same chromosome was $\frac{7}{0}$ relocated in the SEM after photography in the LM. $\stackrel{+}{\mathbb{D}}$ The boundary of the secondary constriction was demarcated by a prominent circumferential groove@ (fig 2d, white arrow) and the heterochromatin 


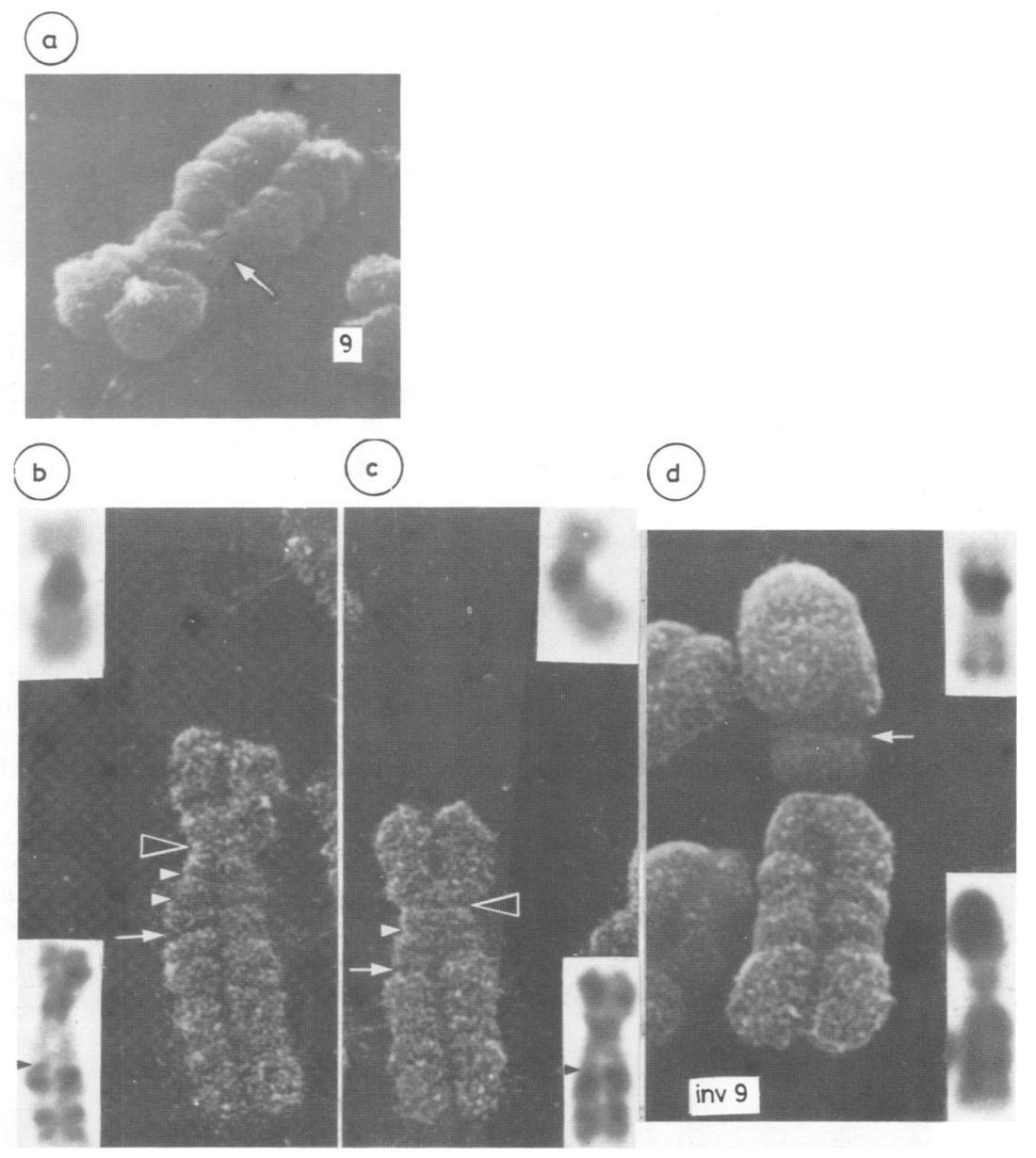

FIG 2 (a) A chromosome 9 viewed in the SEM, upside down. The constricted centromeric region is clearly demonstrated, demarcated by a circumferential groove (white arrow). (Original magnification $\times 8500$.) (b) and (c) A homologous pair of chromosomes 9 viewe firstly in the LM after G banding (lower insets) then in the SEM.A LM C banded pair (prepared in parallel) is shown (upper insets) ; . . nu $n$ the $9 q u \cdot-i(b)$ shows extra $C$ banded material at the centromere when compared to the smaller 9 (c). The centromeric region stains negatively after $G$ banding in the $L M$ (lower insets) and the extent of this region is demarcated by the proximal boundary of $G$ band $9 q 21$ (black arrowheads) corresponding to a prominent circumferential groove in the SEM (white arrows). The centromeres are clearly defined in the SEM (black on white arrowheaas). Within the heterochromatic region circumferential grooves are observed (white arrowheads). (Original magnification $\times 6000$.) (d) This shows an inv (9) detected by $C$ banding (upper inset). This $C$ banded region stains negatively after $G$ banding in the LM (lower inset). When the same chromosome is viewed in the SEM, this region is constricted in appearance and demarcated by a prominent circumferential groove (white arrow). This inversion is complete as no chromatin of constricted morphology is observed on the long arm of this chromosome. (Original magnification $\times$ 10 000.)

showed a constricted morphology (fig 2d), as observed in the non-inverted chromosomes 9 (fig $2 \mathrm{~b}$ and c). Inv (9) may show partial or complete inversion of the heterochromatin. LM C banding studies indicated a complete inversion in this subject (fig 2d, upper inset). This was confirmed by SEM studies in which the chromatin of constricted morphology, related to the heterochromatin, was observed in the short arm and was completely absent from the long arm in all metaphases examined (see fig $2 \mathrm{~d}$ ).

\section{Chromosome 16}

A variable paracentric secondary constriction at the proximal long arm of chromosome 16 is another common site of a human chromosome 
polymorphism. A person was observed with an enlarged secondary constriction of one of his chromosomes $16(16 \mathrm{qh}+)$ detected as a large positively stained $\mathrm{C}$ band in the LM (fig 3a, lower inset). The $C$ band of the homologous 16 was considerably smaller (fig 3a, upper inset). Although not illustrated here the heterochromatin stained intensely with $G$ banding in a similar manner to chromosome 1. A circumferential groove was demonstrated to demarcate the boundary of the secondary constriction of both the $16 \mathrm{qh}+$ and its homologue (figs $3 \mathrm{a}$ and $\mathrm{b}$, white arrows), as also described in chromosomes 1 and 9. This groove was more clearly defined in the $16 \mathrm{qh}+$ when viewed from one side (fig $3 b$ ) than in the upright position (fig 3a). The heterochromatin was of a constricted morphology, as displayed in the other polymorphic chromosomes. Therefore the extent of the heterochromatic region was also clearly defined in chromosomes 16 .

\section{Y chromosome}

The distal portion of the long arm of the $Y$ chromosome stains very darkly with $\mathrm{C}$ banding techniques (fig 4a, lower inset). This region is highly polymorphic, varying greatly in length and amount of heterochromatin. Fig 4 shows an average sized $Y$, chromosome in the LM ((a) upper inset) and an elongated $\mathrm{Y}$ with an increased amount of heterochromatin $(\mathrm{Yqh}+)$ ((b) upper inset). After G

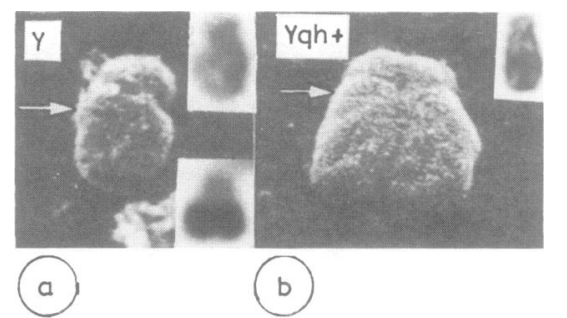

FIG 4 (a) An average sized Y chromosome observed in the $\vec{\omega}$ $S E M$. The distal end of the $Y$ stains positively after $C$ banding (lower inset). (b) Yqh+ observed in the SEM. After $G$ banding a faint positive band demarcates the extent of the heterochromatin in the LM (upper insets) corresponding to a circumferential groove in the SEM (white arrows). (Original magnification $\times 7000$.)

banding a faint $G$ positive band was observed, $\rightarrow$ corresponding in position to the limit of the hetero- $T$ chromatic region, at the boundary between $\mathrm{G}$ bands을 Yq11 and Yq12. ${ }^{31}$ This is shown more clearly in figo $4 \mathrm{~b}$ (inset). From examination of numerous metaph $=$ ases in the SEM this $\mathrm{G}$ band was found to correlate $\overrightarrow{0}$ with a circumferential groove in both chromatidsor (fig $4 \mathrm{a}$ and $\mathrm{b}$, white arrows). Although less pronounced than those grooves corresponding to theo boundaries of the paracentric secondary constrictions, this circumferential groove in the $\mathrm{Y}$ long armo provided a precise definition of the extent of the heterochromatic region.

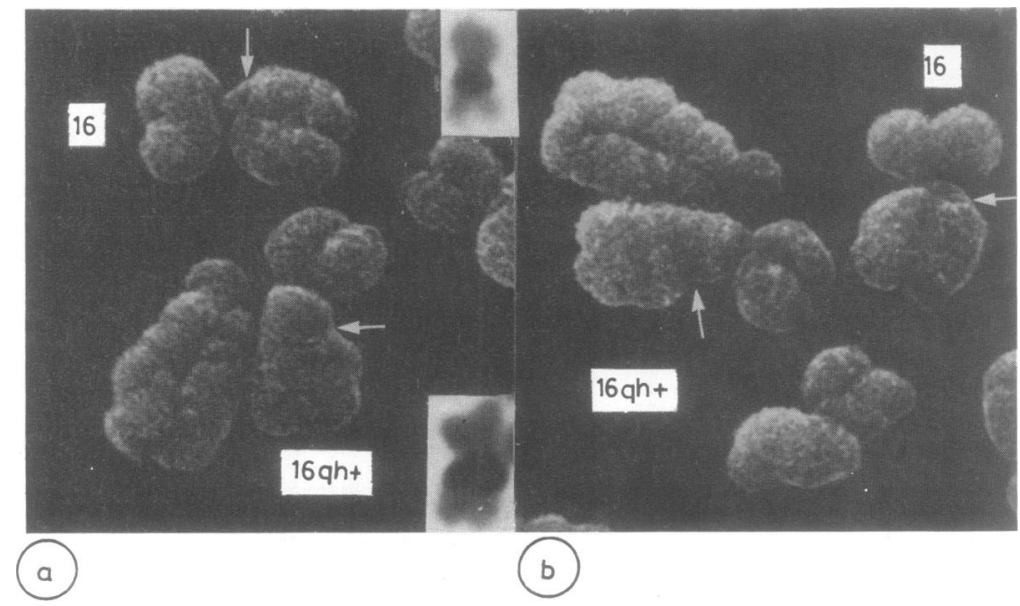

FIG 3 A homologous pair of number 16 chromosomes observed in the SEM. Extra heterochromatic material is revealed in $16 q h+$ by $C$ banding in the LM.(lower inset) when compared to the normal 16 (upper inset). The extent of the heterochromatic region is demarcated by a circumferential groove in the normal 16 and 16 qh + (white arrows). (a) This shows 16qh + in an upright position with the normal 16 on one side. (b) This shows the same field of view at a different angle, with the normal 16 in an upright position and $16 q \mathrm{~h}+$ on one side. When viewed from the side the circumferential $\mathbb{D}$ grooves are more clearly defined (white arrows). (Original magnification $\times 7000$.) 
paracentromeric regions of chromosomes 1,9 , and 16 , or at the distal end of the Y chromosome were selected initially by LM C banding, followed by detailed investigation of $\mathrm{G}$ banded preparations in the SEM.

In each case, $1 \mathrm{qh}+, 9 \mathrm{qh}+$, inv (9), and $16 \mathrm{qh}+$, the positively stained heterochromatic material, was of constricted morphology when compared to the euchromatic regions. This indicated a difference in gross morphology between hetero- and euchromatin produced, possibly, as a result of alternative methods of coiling during compaction into the metaphase chromosome. This has been implicated by several authors from LM observations. ${ }^{32-35}$

The extent of the paracentromeric heterochromatic region of the polymorphic chromosomes was, in each case, defined by a prominent circumferential groove in the SEM. The centromere was also always clearly defined in the SEM. Demarcation of the heterochromatin at the distal end of the Y chromosome was provided by a faint circumferential groove. These observations were not dependent on the uptake of stain. In contrast, in the LM, Giemsa staining was necessary to demonstrate these positive $\mathrm{C}$ bands and the degree of staining was found to be highly variable between cells. ${ }^{25}$ Therefore, an accurate comparative assessment of the extent of the heterochromatic regions between homologous chromosome pairs and between persons is possible using the SEM.

In the inv (9), the same morphological characteristics were observed, and were found to be useful in determination of partial or complete inversion of the heterochromatic region.

Circumferential grooves were observed within the heterochromatin of chromosome 9 , and the number of grooves present reflected the size of the region. For example, the 9qh+, described here, showed two intraheterochromatic grooves and the smaller 9 showed one. This difference in number of grooves was consistently seen in a large number of metaphases from the same person. In our samples no variable staining regions, which might relate to the pattern of intraheterochromatic grooves, were observed in the LM. Other authors have reported the presence of subdivisions within the $\mathrm{C}$ bands (reviewed by Erdtmann ${ }^{25}$ ), but an intercellular variation in number was usually seen. ${ }^{36}$ Therefore, the variation in number of intraheterochromatic grooves observed in the SEM provides a more accurate assessment of relative amounts of heterochromatic material than LM evaluations. To date, grooves have not been observed within the heterochromatic region of chromosomes 1,16 , and $\mathrm{Y}$. The restricted presence of grooves within the polymorphic region of chromosome 9 , and the unique staining ability of the chromosome 9 centromeric region with Giemsa $11,{ }^{37}$ suggests a unique morphology or mode of compaction in this chromosome.

The satellites and short arms of acrocentric chromosomes vary greatly in size and morphology. To demonstrate these differences in the LM sequential and diverse staining is required. ${ }^{34} 3839$ These techniques may reduce the definition of the satellites, owing to variation in the uptake of stain. Therefore, the appearance may be variable from cell to cell, and only gross changes in morphology may be accurately assessed in the LM. After G banding, the individual acrocentric chromosomes were readily identified in the SEM. Also, the morphology of the satellites was consistently maintained from metaph- $\vec{\sigma}$ ase to metaphase, since observation by SEM does 은 not depend on the presence of stain. ${ }^{30}$ Three dimensional viewing from different angles in the $T$ SEM, as demonstrated here, provides the opportunity for a direct comparison of size or volume of satellites to be made. In addition small morphological variations of the short arms, or within the $\overrightarrow{0}$ centromeric regions, of the acrocentric chromo- of somes were easily defined by SEM when definition was uncertain in the LM.

The increased detail of the chromosome polymorphisms that are observed by high resolution SEM reveals the scope of this technique for application to clinical cases, for example, in those cases where it is important to know the parental origin of chromosome polymorphisms. These may include the observation of a female karyotype from chorionic villi sampling at 8 or 10 weeks' gestation. Increased ability to recognise parental polymorphisms in the fetal cells may be of use in exclusion of maternal cell contamination of cultures. Also, the high resolution investigation of polymorphisms may be important in identification of the parental origin of the extra chromosome 21 in Down's syndrome in those cases where no obvious markers were detected by LM techniques.

We thank the Department of Medical Genetics, St $N$ Mary's Hospital, Manchester for providing the specimens and for their cytogenetic advice. This $\mathrm{N}$ work was supported by MWRHA project grant $418 \omega$ and the Cancer Research Campaign.

\section{References}

1 Hansmann I. Structural variability of human chromosome 9 in relation to its evolution. Hum Genet 1976;31:247-62.

2 Magenis RE, Palmer CG, Wang L, et al. Heritability of chromosome banding variants. In: Hook EB, Porter IH, eds. Population cytogenetics. New York: Academic Press, 1977:179 88. 
${ }^{3}$ Hoehn H, Kam A, Karp LE, Martin GM. Somatic stability of variant C-band heterochromatin. Hum Genet 1977:35:163-8.

${ }^{4}$ Robinson JA, Buckton KE, Spowart G, et al. The segregation of human polymorphisms. Ann Hum Genet 1976;40:113-21.

5 Verma RS, Dosik H. Human chromosomal heteromorphisms: nature and clinical significance. Int Rev Cytol 1980;62:361-83.

${ }^{6}$ Chamberlin J, Magenis RE. Parental origin of de novo chromosome rearrangements. Hum Genet 1980;53:343-7.

${ }^{7}$ Kajii T, Niikawa N. Origin of triploidy and tetraploidy in man: 11 cases with chromosome markers. Cytogenet Cell Genet 1977:18:109-25.

8 Jacobs PA, Mayer M. The origin of human trisomy. A study of heteromorphisms and satellite associations. Ann Hum Genet 1981:45:357-65.

9 Magenis RE, Overton KM, Chamberlin J, Brady T, Lovrien E. Parental origin of the extra chromosome in Down's syndrome. Hum Genet 1977;37:7-16.

${ }^{10}$ Mikkelsen M, Pousen H, Grinsted J, Lange A. Non-disjunction in trisomy 21: study of chromosomal heteromorphisms in 110 families. Ann Hum Genet 1980:44:17.

${ }^{11}$ Robinson JA. Origin of extra chromosome in trisomy 21. Lancet $1973 ; \mathbf{i}: 131-3$.

12 Uchida IA. Paternal origin of the extra chromosome in Down's syndrome. Lancet 1973;ii:1258.

13 de la Chapelle A, Fellman J, Unnerus V. Determination of human paternity from the length of the Y chromosome. Ann Genet (Paris) 1967;10:60-4.

14 Olson SB, Magenis RE, Rowe SI, Lovrien EW. Chromosome heteromorphism analysis in cases of disputed paternity. Am J Med Genet 1983;15:47-55.

15 Jacobs PA, Frackiewicz A, Law P, Hilditch CJ, Morton NE. The effect of structural aberrations of the chromosomes on reproductive fitness in man. Clin Genet 1975;8:169-78.

16 Jacobs PA. Human chromosome heteromorphisms. In: Steinberg AG, Bearn AG, Motulsky AG, Childs B, eds. Progress in Medical Genetics. Vol 2. Saunders: Philadelphia, 1977:251-74.

${ }^{17}$ Boué J, Taillemite JL, Hazael-Massieux P, Leonard C, Boué A. Association of pericentric inversion of chromosome 9 and reproductive failure of ten unrelated families. Hum Genet 1975;30:217-24.

${ }^{18}$ Holbek S, Friedrich U, Lauritsen JG. Therkelsen AJ. Marker chromosomes in parents of spontaneous abortuses. Humantenetik 1974:61-4.

${ }_{19}$ Halbrecht I, Shabtay F. Human chromosome polymorphism and congenital malformations. Clin Genet 1976;10:113-22.

20 Wang HS, Hamerton JL. C-band polymorphisms of chromosomes 1,9 and 16 in four subgroups of mentally retarded patients and a normal control population. Hum Genet 1979;51:269-75.

21 Atkin NB, Baker MC. Abnormal chromosomes and number 1 heterochromatin variants revealed in C-banded preparations from 13 bladder carcinomas. Cytobios 1977;18:101-9.

${ }^{22}$ Atkin NB, Baker MC. Pericentric inversion of chromosome 1.
Frequency and possible association with cancer. Cytogenet Cell Genet 1977;19:180-9.

23 Atkin NB, Pickthall VJ. Chromosomes 1 in 14 ovarian cancers: heterochromatin variants and structural changes. Hum Genet 1977;38:25-33.

${ }^{24}$ Petkovic I. Constitutive heterochromatin of chromosomes 1, 9 and 16 in 90 patients with malignant disease and 91 controls. Cancer Genet Cytogenet 1983;8:151-8.

${ }^{25}$ Erdtmann B. Aspects of evaluation, significance and evolution of human C-band heteromorphism. Hum Genet 1982;61:281-94.

${ }^{26}$ Sumner AT. A simple technique for demonstrating centric heterochromatin. Exp Cell Res 1972;75:304-6.

27 Seabright M. A rapid banding technique for human chromosomes. Lancet 1971;ii:971-2.

${ }^{28}$ Drury RAB. Wallington EA. Carleton's histological technique. Oxford: Oxford University Press, 1980:495.

${ }^{29}$ Harrison CJ, Britch M. Allen TD, Harris R. Scanning electron microscopy of the G-banded human karyotype. Exp Cell Res 1981:134:141-54.

30) Harrison CJ, Allen TD, Britch M, Harris R. High resolution scanning electron microscopy of human metaphase chromosomes. J Cell Sci 1982:56:409-22.

${ }^{31}$ ISCN (1981). An international system for human cytogenetic nomenclature: high resolution banding. Cytogenet Cell Genet 1981:31:1-23.

${ }^{32}$ Madan K, Bobrow M. Structural variation in chromosome No 9. Ann Genet (Paris) 1974;17:81-6.

${ }^{33}$ Schmiady H, Sperling K. Length of human C-bands in relation to the degree of chromosome condensation. Hum Genet 1976:49:243-50.

${ }^{34}$ Bălicek P, Žižka J, Skalska $\mathrm{H}$. Length of human constitutive heterochromatin in relation to chromosomal contraction. Hum Genet 1977;38:189-93.

35. Selezneva TG, Deryaguin GV, Badaev NS, Prokof jevaBel'govskaya AA. The analysis of eu- and heterochromatin spiralization dynamics in heteromorphic homologous chromosomes No 1 in man. Cytologia (Tokyo) 1977:3:298-302.

36 Wegner H, Pawlowitzki IH. Quantification of C-band polymorphism by centromeric elevations (ce-bands). Hum Genet 1981;58:302-5.

${ }^{37}$ Bobrow M, Madan K, Pearson PL. Staining of some specific regions of human chromosomes, particularly the secondary constriction of No 9. Nature 1972:238:122-4.

${ }^{3 *}$ Chen TR, Kao ML, Marks J, Chen YY. Polymorphic variants in human chromosome 15. Am J Med Genet 1981;9:61-6.

${ }^{39}$ Wachtler F, Musil R. On the structure and polymorphism of the human chromosome no 15. Hum Genet 1980;56:115-8.

Correspondence and requests for reprints to Dr C J Harrison, Department of Cell Biology and Cytogenetics, Paterson Laboratories, Christie Hospital and Holt Radium Institute, Manchester M20 9BX. 